\title{
Sildenafil and FDP-Sr attenuate diabetic cardiomyo- pathy by suppressing abnormal expression of myo- cardial CASQ2, FKBP12.6, and SERCA2a in rats
}

\author{
Yu-si CHENG ${ }^{1}$, De-zai DAI ${ }^{1, *}$, Hui $\mathrm{JI}^{1, *}$, Qi ZHANG ${ }^{2}$, Yin DAI ${ }^{1}$ \\ ${ }^{1}$ The Faculty of Pharmacy, China Pharmaceutical University, Nanjing 210009, China; ${ }^{2}$ The Bioengineering and Pharmaceutical Faculty, \\ Nanjing University of Technology, Nanjing 210009, China
}

\begin{abstract}
Aim: To study whether calcium-modulating proteins CASQ2, FKBP12.6 and SERCA2a participate in diabetic cardiomyopathy, and whether the beneficial actions of testosterone, sildenafil or fructose diphosphate Sr (FDP-Sr) in the treatment of diabetic cardiomyopathy result from suppressing these molecules.

Methods: Fifty male Sprague-Dawley (SD) rats were divided into five groups. Except for the normal group (non-diabetic), the other four groups were injected with streptozotocin (STZ, $60 \mathrm{mg} / \mathrm{kg}$, ip) to induce diabetes. Four weeks after STZ injection, the four groups received sildenafil (12 $\mathrm{mg} \cdot \mathrm{kg}^{-1} \cdot \mathrm{d}^{-1}$, ig, for 4 week), FDP-Sr (200 mg/kg, ig, for 4 week), testosterone propionate $\left(4 \mathrm{mg} \cdot \mathrm{kg}^{-1} \cdot \mathrm{d}^{-1}\right.$, sc, for 4 week), or no treatment, respectively.

Results: In the diabetic rats, blood glucose, free fatty acids, triglycerides, total cholesterol, and low-density lipoprotein cholesterol (LDL-C) were significantly increased, while high-density lipoprotein cholesterol (HDL-C) was significantly reduced, as compared to the non-diabetic rats. Cardiac dysfunction and myocardial hypertrophy of the diabetic rats were associated with increased mRNA and protein expression of iNOS, OBRb, and PKCE, while expression of CASQ2, SERCA2a, and FKBP12.6 was significantly down-regulated. Sildenafil and FDP-Sr, but not testosterone, significantly attenuated the biomarker abnormalities, without changing the metabolic abnormalities.

Conclusion: CASQ2, FKBP12.6 and SERCA2a were down-regulated in diabetic cardiomyopathy. Sildenafil and FDP-Sr, but not testosterone, attenuated the cardiac dysfunction in diabetic cardiomyopathy, without changing the metabolic abnormalities, which may results from inhibiting oxidative and inflammatory cytokines and improving calcium homeostasis.
\end{abstract}

Keywords: diabetic cardiomyopathy; CASQ2; FKBP12.6; SERCA2a; sildenafil; FDP-Sr; testosterone; calcium homeostasis

Acta Pharmacologica Sinica (2011) 32: 441-448; doi: 10.1038/aps.2010.226; published online 28 Mar 2011

\section{Introduction}

By 2025, there will be an estimated 300 million people worldwide suffering from diabetes mellitus (DM) ${ }^{[1]}$. Up to $80 \%$ of morbidity and mortality in diabetic patients is due to cardiovascular disease (CVD). Initially, cardiac changes are asymptomatic. Later, left ventricular hypertrophy as well as diastolic and systolic dysfunction develops, and finally, symptomatic heart failure occurs. This unique pathological process is referred to as diabetic cardiomyopathy ${ }^{[2,3]}$.

The main cause of diabetic cardiomyopathy is metabolic changes characterized by a sustained elevation of blood glucose and lipids, which promotes the formation of advance glycation end products (AGEs) over time ${ }^{[4,5]}$. AGE excess

\footnotetext{
* To whom correspondence should be addressed.

E-mail dezaidai@vip.sina.com; huijicpu@163.com

Received 2010-08-24 Accepted 2010-12-17
}

stimulates glycosylation, which adversely affects cardiac contractility, likely through calcium-modulating proteins in the sarcoplasmic reticulum (SR). Sarcoplasmic reticulum calcium pump 2a (SERCA2a) is responsible for the uptake of intracellular calcium back into the SR and is essential for cardiac function. SERCA2a is very sensitive to posttranslational modification, and glycosylation of SERCA2a leads to cardiac dysfunction $^{[6,7]}$. The systolic function of myocardial cells relies mainly on ryanodine receptor type 2 (RyR2), FOK binding protein/ calstabin 2 (FKBP12.6), SERCA2a, and troponin C, whereas diastolic function is dependent on SERCA2a and sodium calcium exchangers (NCX). Calcium homeostasis, the basis of cardiac function, relies on the release (RyR2 and FKBP12.6), uptake (SERCA2a and phospholamban, PLB), and storage (calsequestin 2, CASQ2) of $\mathrm{Ca}^{2+}$ in the SR. RyR2, FKBP12.6, SERCA2a, and PLB were found to be downregulated in failing hearts and diabetic cardiomyopathy, but these changes can be 
alleviated by drug intervention ${ }^{[8,9]}$. Furthermore, SERCA2a overexpression is protective against STZ-induced cardiotoxicity in rats ${ }^{[10]}$. CASQ2 holds $\mathrm{Ca}^{2+}$ in the ER lumen and affects calcium release during the cardiac cycle. CASQ2 or RyR2 mutations may lead to a dangerous arrhythmia called catecholaminergic polymorphic ventricular tachycardia (CPVT) ${ }^{[11]}$. Additionally, abnormal CASQ2 expression can be caused by isoproterenol and relieved by CPU0213, an endothelin receptor antagonist ${ }^{[11,12]}$. We aimed to explore if downregulation of CASQ2 disrupts calcium homeostasis in diabetic cardiomyopathy. Currently, no studies have investigated how changes in CASQ2 expression affect diabetic cardiomyopathy.

Recent evidence has suggested that low serum testosterone, as seen in male hypogonadism, is an independent risk factor for cardiovascular disease (CVD) due to increased inflammatory factors and cytokines in the myocardium ${ }^{[13]}$. Testosterone replacement therapy (TRT) has become an attractive intervention that may relieve the cardiac dysfunction associated with late-onset hypogonadism, a potential oxidative stress, through its antioxidant activity ${ }^{[14-16]}$.

Sildenafil, a phosphodiesterase type 5 (PDE5) selective inhibitor, has been shown to improve heart failure by correcting vascular dysfunction through increasing vascular cyclic guanosine monophosphate (cGMP), endogenous NO, and free radical-scavenging activity ${ }^{[17,18]}$. However, the efficacy of sildenafil in attenuating diabetic cardiomyopathy has not been shown. FDP-Sr, a derivative of FDP that contains three extra adenosine triphosphates (ATPs) in the moiety, has been shown to be effective in attenuating diabetic cardiomyopathy ${ }^{[19,20]}$ (Figure 1).

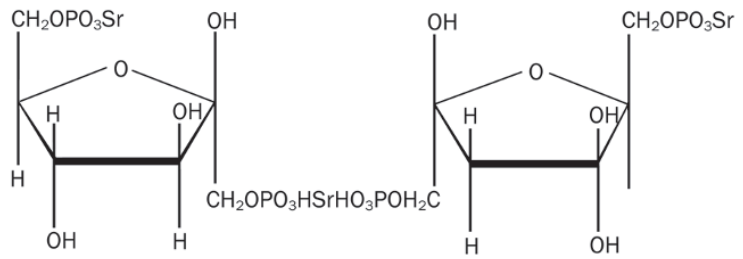

Figure 1. The chemical structure of FDP-Sr.

We hypothesized that diabetic cardiomyopathy was a unique disorder characterized by cardiac dysfunction associated with low serum testosterone and downregulated calciumhandling proteins at the SR, including CASQ2, FKBP12.6, and SERCA2a. In this study, the effects of FDP-Sr, sildenafil, and testosterone on diabetic cardiomyopathy were tested in terms of relieving oxidative stress and normalizing calcium homeostasis in the myocardium.

\section{Materials and methods} Animals

Adult male Sprague-Dawley (SD) rats weighing 200-220 g were used. They were housed in a controlled environment and allowed free access to water and food. Animal handling and experimental procedures were in accordance with the regulations of the Jiangsu Provincial Government and the Principles of Laboratory Animal Care published by the US National Institutes of Health (NIH Publication No 85-23, revised 1996).

\section{Experimental protocol}

Fifty SD male rats were randomly divided into five groups. Except for the non-diabetic group, rats were injected with STZ (60 mg/kg, intraperitoneal) once. On days 7, 14, 21, and 28 after STZ administration, blood glucose was measured. Blood glucose consistently greater than $16.7 \mathrm{mmol} / \mathrm{L}$ was considered to be indicative of diabetes. During weeks 5 to 8 after STZ injection, animals were treated with sildenafil (12 $\mathrm{mg} / \mathrm{kg}$, ig), FDP-Sr (200 mg/kg, ig), or testosterone propionate (4 mg/kg, subcutaneous). Rats in the non-diabetic or STZ untreated groups were given an equal amount of citric acid buffer.

\section{Hemodynamic changes}

On day 57, the rats were anesthetized with urethane (1.5 $\mathrm{g} / \mathrm{kg}$, intraperitoneal). A catheter (PE 50, ID $0.58 \mathrm{~mm}$, OD $0.965 \mathrm{~mm}$, Becton Dickinson, San Jose, CA, USA) was inserted into the left ventricular (LV) chamber through the right carotid artery to measure LV systolic blood pressure (LVSP), LV end diastolic blood pressure (LVEDP), maximum rising rate of $\mathrm{LV}$ pressure $\left(\mathrm{LV}+\mathrm{d} p / \mathrm{d} t_{\max }\right)$, and minimum declining rate of $\mathrm{LV}$ pressure (LV-d $\left.p / \mathrm{d} t_{\min }\right)$, as described previously ${ }^{[21]}$.

\section{LV weight index}

After hemodynamic measurements, the rats' hearts were excised and dissected into the LV free wall plus septum (LV) and right ventricle (RV). The LV weight index was assessed as the weight of the LV divided by body weight (LVW/BW).

\section{Cell culture}

Cardiomyocytes isolated from neonatal rats (1-3 d old) were cultured for $72 \mathrm{~h}$ in DMEM medium containing $20 \%$ fetal bovine serum and $0.1 \mathrm{mmol} / \mathrm{L}$ bromodeoxyuridine. The adherent cell density was $0.5 \times 10^{6} / \mathrm{mL}$. Then the cardiomyocytes were incubated for $24 \mathrm{~h}$ with testosterone $\left(10^{-5}, 10^{-6}\right.$, or $\left.10^{-7} \mathrm{~mol} / \mathrm{L}\right), \mathrm{FDP}-\mathrm{Sr}\left(10^{-5}, 10^{-6}\right.$, or $\left.10^{-7} \mathrm{~mol} / \mathrm{L}\right)$, or sildenafil $\left(10^{-5}\right.$, $10^{-6}$, or $\left.10^{-7} \mathrm{~mol} / \mathrm{L}\right)$. The control cells were treated with DMSO. CASQ2 mRNA and protein expression were measured to investigate if changes in CASQ2 could be induced by testosterone, FDP-Sr, or sildenafil in the absence of high-glucose medium.

\section{RT-PCR}

Total RNA was extracted using Trizol reagent (Biouniquer Technology Company, Nanjing, China) according to the manufacturer's instructions. Five milligrams of RNA was used to synthesize cDNA using SUPERSCRIPT II RNase H-Reverse Transcriptase (Biouniquer Technology Company, Nanjing, China) according to the manufacturer's protocol. This cDNA was used as a template for the PCR reactions ${ }^{[22]}$. PCR primers specific for iNOS, OBRb, PKC $\varepsilon$, CASQ2, FKBP12.6, SER- 
CA2a, and GAPDH were designed as indicated in previous reports $^{[12,21]}$.

\section{Western blotting}

For quantitative analysis of myocardial phosphorylated PKC $\varepsilon$ (pPKC $\varepsilon$ ), CASQ2, FKBP12.6, and SERCA2a protein levels, 100 $\mathrm{mg}$ of heart tissue was homogenized in $1 \mathrm{~mL}$ of extraction buffer and centrifuged at $10000 \times \mathrm{g}$ for $10 \mathrm{~min}$ as previously described $^{[21]}$. After determining protein concentration, the supernatants were stored at $-20^{\circ} \mathrm{C}$. An aliquot was heated to $100{ }^{\circ} \mathrm{C}$ for $10 \mathrm{~min}$ and size fractionated using $10 \%$ sodium dodecyl sulfate-polyacrylamide gel electrophoresis (SDSPAGE). The extracted protein was transferred to a nitrocellulose membrane and blocked with nonfat milk $(5 \% w / v)$ followed by incubation with primary antibody for another $1-2 \mathrm{~h}$ at $37^{\circ} \mathrm{C}$. After three washes, the blot was incubated with horseradish peroxidase-conjugated goat secondary antibody IgG (Affinity Bioreagents; 1:500) for an additional $1 \mathrm{~h}$ at $37^{\circ} \mathrm{C}^{[22]}$. Antigen was detected with a DAB kit. The density of the bands was analyzed using Labworks imaging acquisition and analysis software (GDS8000, Syngene, UK).

\section{Statistical analysis}

SPSS 11.5 (SPSS company, Chicago, USA) was used to analyze the results. Data were presented as the mean $\pm S D$. For statistical evaluation, one-way analysis of variance was used followed by Dunnett's test. The Student Newman Keuls test was performed when the variance was equal, and the GamesHowell test was performed when the variance was not equal. A $P$-value of less than 0.05 was considered statistically significant.

\section{Results}

Serum glucose, lipids, and androgens

In the diabetic group, blood glucose, free fatty acids, triglycerides, total cholesterol, and LDL-C were significantly increased, and HDL-C was significantly decreased, indicating that the STZ-injected rats had marked metabolic changes $(P<0.01)$. Single treatment with testosterone, Sildenafil, and FDP-Sr had no effects on the metabolic changes (Figure 2). Serum androgens $(\mathrm{nmol} / \mathrm{L})$ were sharply reduced in the STZ-injected rats $(4.6 \pm 1.9, P<0.01)$ compared to the non-diabetic group (15.6 \pm 4.1$)$. Serum androgens were significantly elevated in the testosterone group compared to the diabetic untreated group $(12.9 \pm 2.1, P<0.01)$. There was no difference compared to the non-diabetic rats. The FDP-Sr group had a moderate increase in serum androgen compared to the diabetic untreated group $(10.6 \pm 2.6, P<0.01)$. Sildenafil did not raise serum androgen (5.8 \pm 2.1$)$.

\section{Cardiac performance}

In the diabetic group, LVSP and LV plus $\mathrm{d} p / \mathrm{d} t_{\max }$ decreased significantly, while LVEDP and LV-d $p / \mathrm{d} t_{\min }$ significantly increased compared to the non-diabetic group $(P<0.01)$. Sildenafil and FDP-Sr led to a significant recovery in cardiac dysfunction $(P<0.01)$. Additionally, the diabetic untreated group had a significantly increased LV mass index compared to the normal group $(P<0.01)$. This increase was blunted by FDP-Sr and sildenafil. In contrast, testosterone had no effect on the hemodynamic or LV mass index abnormalities (Figure 3).
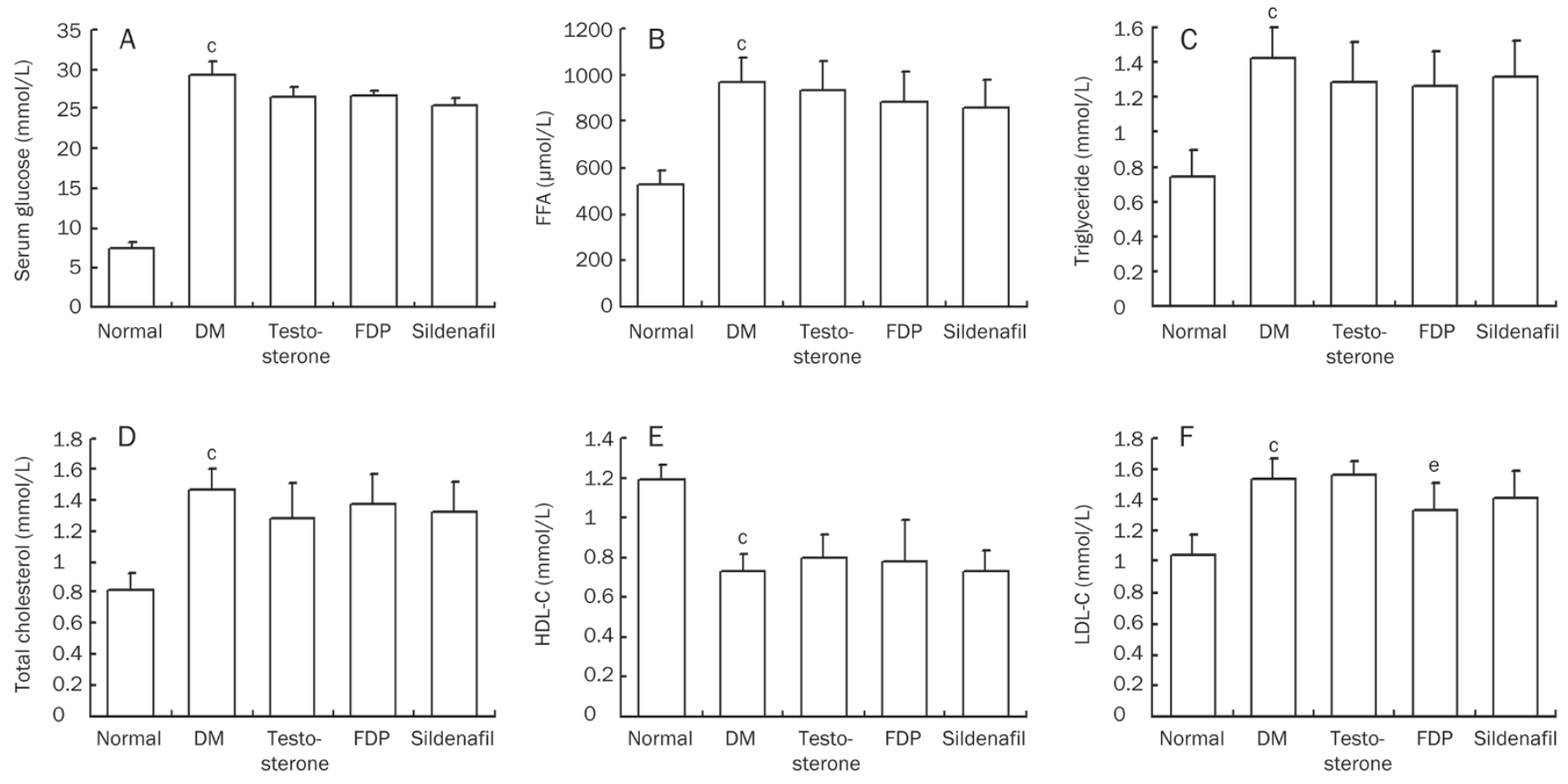

Figure 2. Changes in blood sugar and lipid were found in STZ-injected rats. The metabolic changes in DM rats were significant and testosterone propionate, sildenafil, and FDP-Sr had no effects on the altered sugar and lipid metabolism. (A) Serum glucose; (B) Free fatty acid (FFA); (C) Triglyceride; (D) Total cholesterol; (E) HDL-C; (F) LDL-C. Mean \pm SD. $n=10$. ${ }^{\circ} P<0.01$ vs normal. ${ }^{\mathrm{e}} P<0.05$ vs DM. 

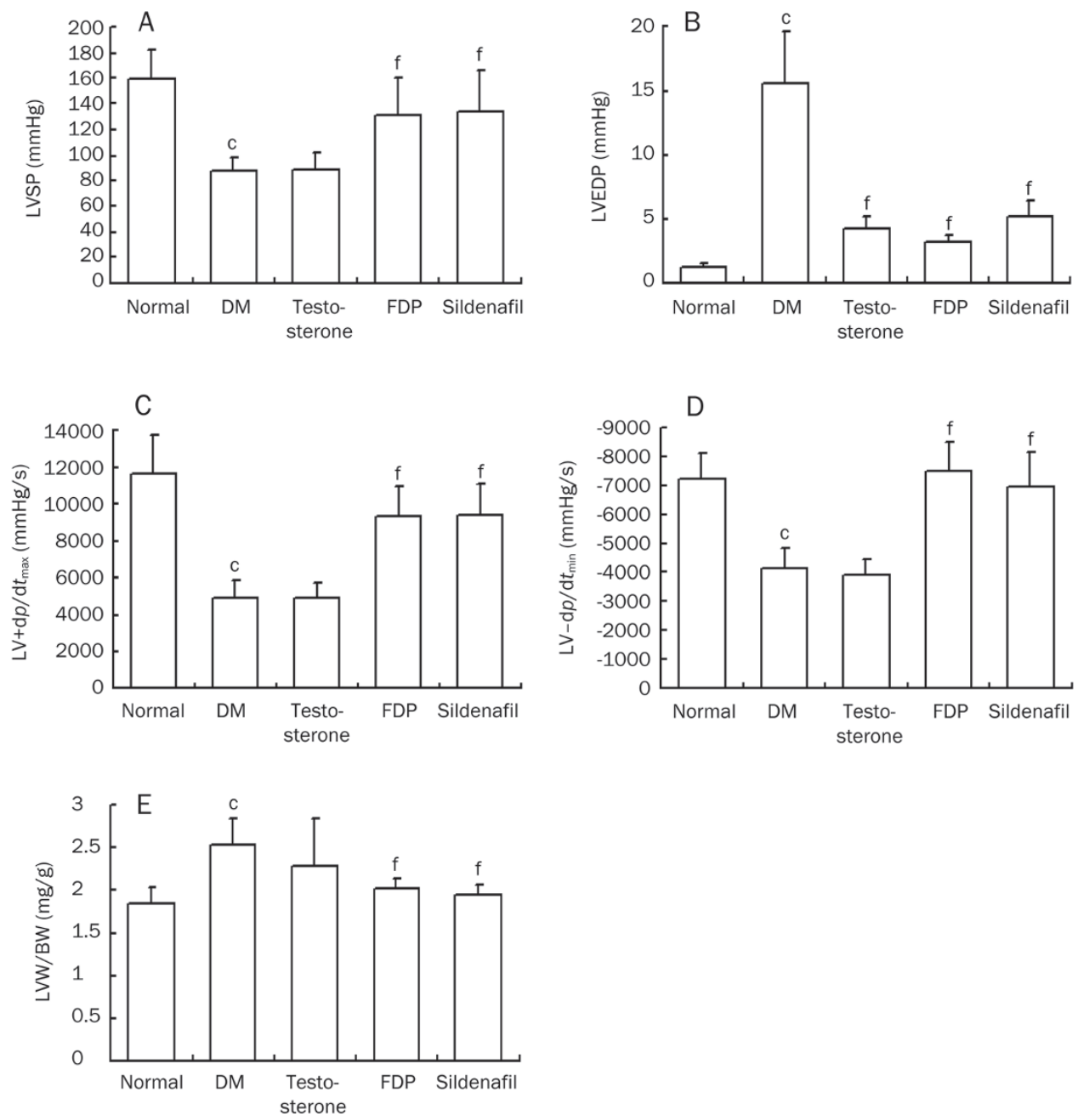

Figure 3. FDP-Sr and sildenafil improved hemodynamic parameters and heart weight index in STZ injected rats. (A) LVSP; (B) LVEDP; (C) LV+dp/dt max $_{\text {; }}$; (D) $\mathrm{LV}-\mathrm{dp} / \mathrm{d} t_{\min }$; (E) the LV weight index. Mean \pm SD. $n=10 .{ }^{\circ} P<0.01$ vs normal; ${ }^{\mathrm{f}} P<0.01$ vs DM.

\section{Oxidative stress}

Plasma malondialdehyde (MDA), a measure of oxidants, was significantly elevated, and GSH-Px, an antioxidant marker, was significantly decreased in the diabetic untreated group compared to the non-diabetic group ( $P<0.01$, respectively). Therefore, the diabetic rats had significant oxidative stress. Sildenafil and FDP-Sr suppressed MDA and raised GSH-PX levels compared to the diabetic untreated group $(P<0.01$, Figure 4). Thus, the antioxidant activity of sildenafil and FDP-Sr ameliorated the pathological changes in diabetic cardiomyopa- thy. Testosterone did not significantly block these pathological changes (Figure 4).

\section{Cardiac iNOS, OBRb, and PKC $\varepsilon$}

In the diabetic rats the mRNA expression of iNOS and OBRb, a leptin receptor, was significantly increased $(P<0.01)$. Additionally, PKC $\varepsilon$ mRNA expression was significantly increased $(P<0.01)$. Sildenafil and FDP-Sr ameliorated the abnormal expression of iNOS, OBRb, and PKCE mRNA $(P<0.01)$. In contrast, testosterone did not affect any of these biomarkers
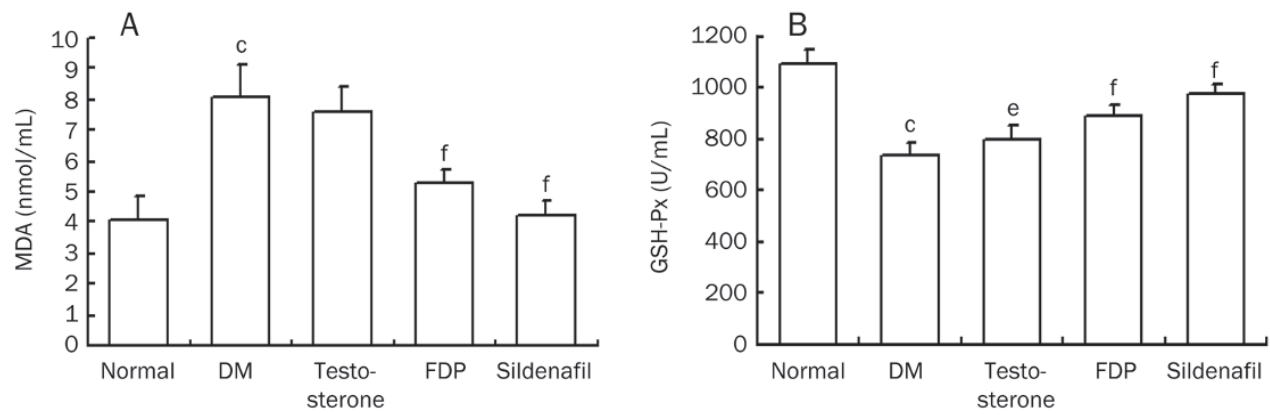

Figure 4. Changes of MDA and GSH-PX in serum were improved by FDP-Sr or sildenafil in diabetic rats. (A) MDA; (B) GSH-Px. Mean \pm SD. $n=10 .{ }^{c} P<0.01$ vs normal; ${ }^{\mathrm{e}} P<0.05,{ }^{\mathrm{f}} P<0.01$ vs DM. 
except iNOS mRNA (Figure 5).

\section{Calcium homeostasis}

Calcium homeostasis in the myocardium is maintained by the calcium-handling proteins, which control intracellular calcium release, uptake, and storage in the SR and are manipulated by bioactive molecules, including FKBP12.6, the main regulator of RyR2, as well as SERCA2a and CASQ2. In the diabetic rats, mRNA and protein expression of CASQ2, SERCA2a, and FKBP12.6 were reduced $(P<0.01)$. This indicates that aspects of calcium homeostasis, such as calcium release, uptake during diastole, and calcium-storing capacity in the SR are impaired in diabetic cardiomyopathy. The biomarker abnormalities may lead to heart failure and severe arrhythmias, which are likely to appear in diabetic cardiomyopathy. Sildenafil and FDP-Sr attenuated the abnormal expression of these molecules $(P<0.01)$. Testosterone ameliorated CASQ2 and SERCA2a mRNA expression but had no effects on their protein expression. Testosterone had no effects on both mRNA and protein expression of FKBP12.6 (Figure 6).

CASQ2 mRNA and protein expression were measured in cardiomyocytes grown in normal medium with normal glucose as well as sildenafil, FDP-Sr, and testosterone. No difference in CASQ2 expression was found in these cells compared to the cardiomyocytes in the absence of drug interventions (Figure 7). Therefore, we believe that sildenafil and FDP-Sr are effective in improving abnormal calcium homeostasis and cardiac dysfunction by suppressing the pathological processes due to hyperglycemia in the diabetic rats.

\section{Discussion}

In diabetic cardiomyopathy, low serum testosterone and changes in glucose and lipid metabolism are the main factors responsible for disease progression. The cardiac dysfunction and hypertrophy in diabetic rats responded significantly to sildenafil and FDP-Sr but not testosterone. However, sildenafil and FDP-Sr did not improve hyperglycemia or hypercholesterolemia. The metabolic changes were not linked directly to cardiac dysfunction. Intermediate events, such as ROS and inflammatory factors, are critical in the pathogenesis of diabetic cardiomyopathy.

In this study, cardiac dysfunction in rats likely correlates with low serum testosterone; thus, we treated the mice with exogenous testosterone, which is a common way to correct this deficiency ${ }^{[23]}$. FDP-Sr moderately raises serum testosterone, likely through improving pathological changes in the testes. In contrast, sildenafil plays no role in correcting low serum testosterone. As a result, sildenafil and FDP-Sr, rather than testosterone supplement, dramatically attenuate cardiac dysfunction and oxidative stress. Exogenous testosterone did not alleviate diabetic cardiomyopathy, likely because it lacks significant antioxidant activity and cannot overcome the pathological changes induced by hyperglycemia.

Increased oxidative stress in vivo has been shown to contribute to the pathogenesis of diabetic cardiomyopathy ${ }^{[24,25]}$. MDA, a lipid oxidation end product, affects the mitochondrial respiratory chain complex and the activities of key enzymes in the mitochondria. Excess reactive oxygen species (ROS) come from mitochondria and result from the activities of NADPH
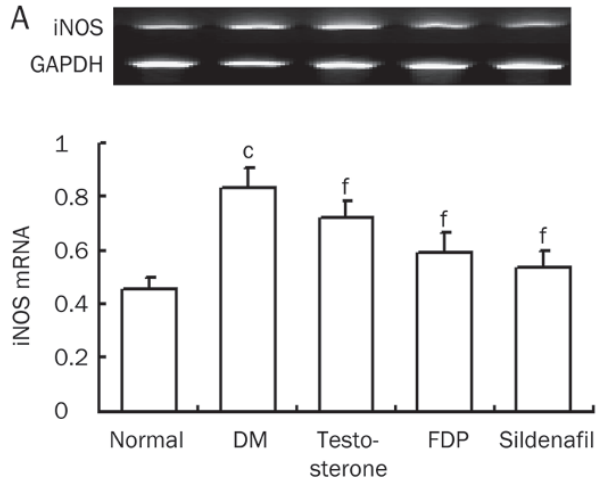

C
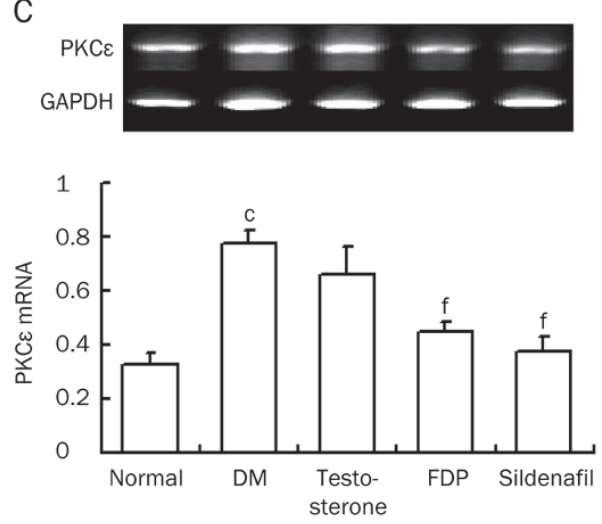
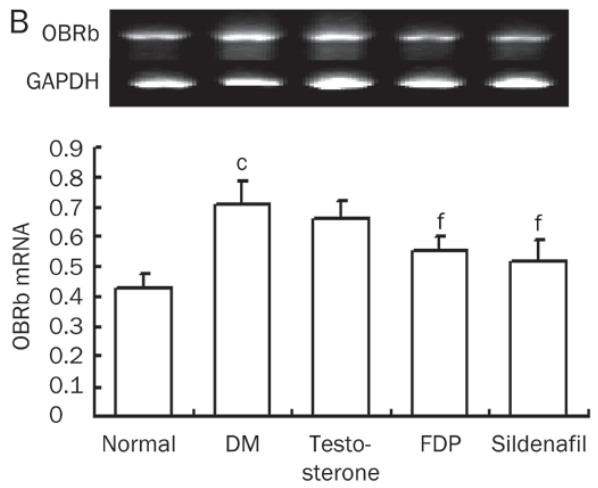

D
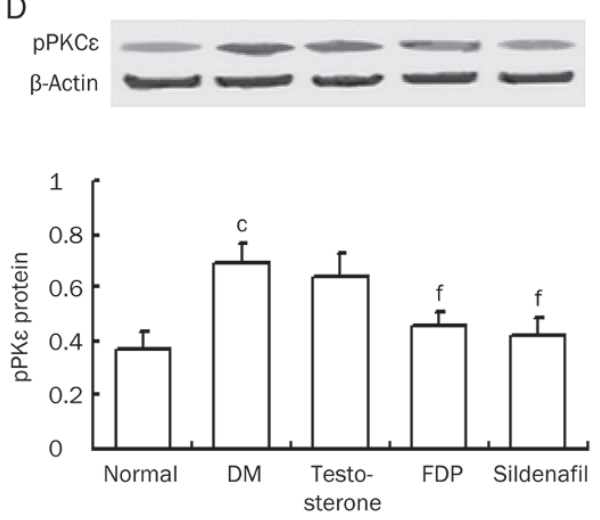

Figure 5. FDP-Sr and sildenafil inhibited abnormal mRNA expression of iNOS, $\mathrm{OBRb}, \mathrm{PKC} \varepsilon$, and protein exprsssion of pPKCE. (A) iNOS mRNA; (B) OBRb mRNA; (C) PKC $\varepsilon$ mRNA; (D) pPKC $\varepsilon$ protein. Mean \pm SD. $n=10 .{ }^{\circ} P<0.01$ vs normal; ${ }^{\mathrm{f}} P<0.01$ vs DM. 
A
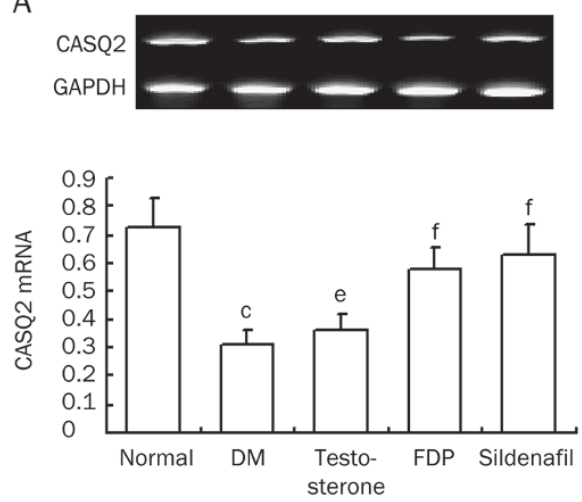

B
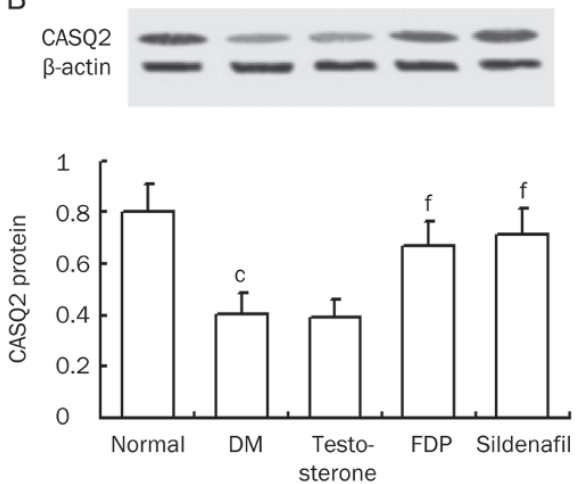

C
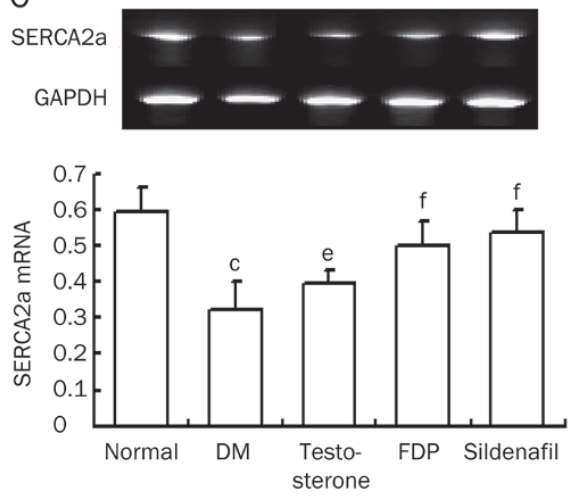

D
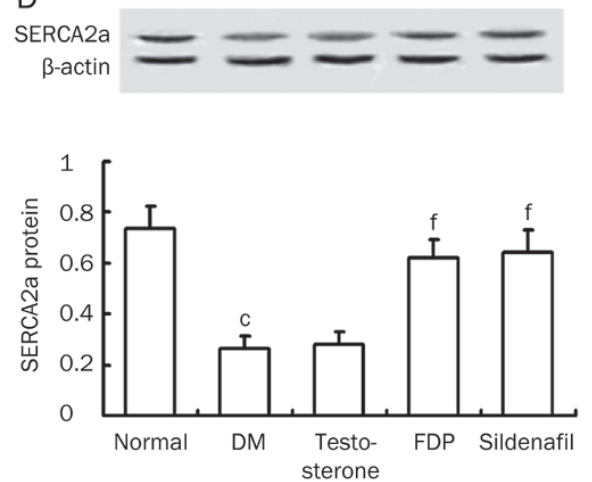

E
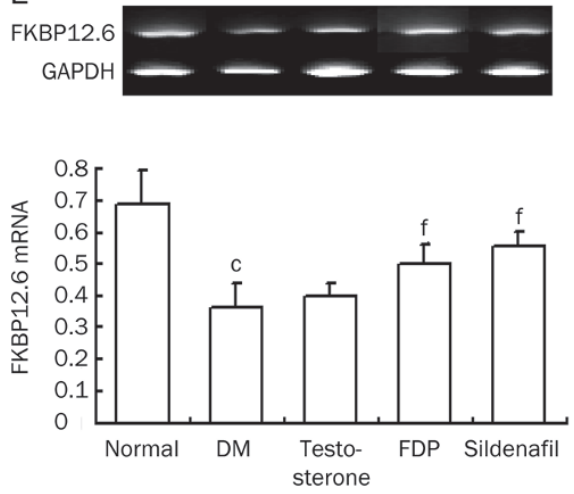

$\mathrm{F}$
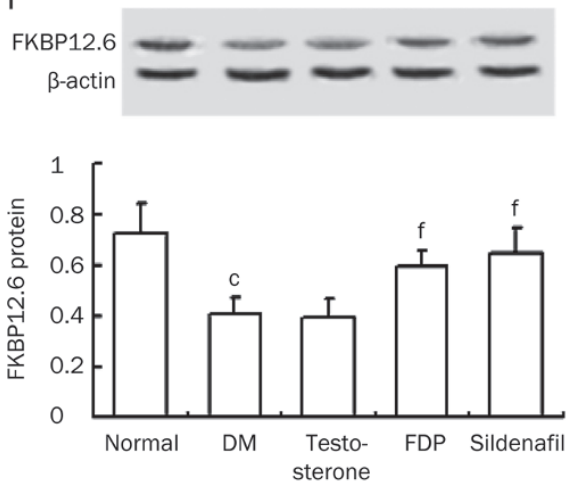

Figure 6. FDP-Sr and sildenafil improved abnormal mRNA and protein expressions of the calcium handing system. (A) CASQ2 mRNA. (B) CASQ2 protein. (C) SERCA2a mRNA. (D) SERCA2a protein. (E) FKBP12.6 mRNA. (F) FKBP12.6 protein. Mean \pm SD. $n=10 .{ }^{b} P<0.05,{ }^{c} P<0.01$ vs normal; ${ }^{\mathrm{e}} P<0.05,{ }^{\mathrm{f}} P<0.01$ vs DM.

A
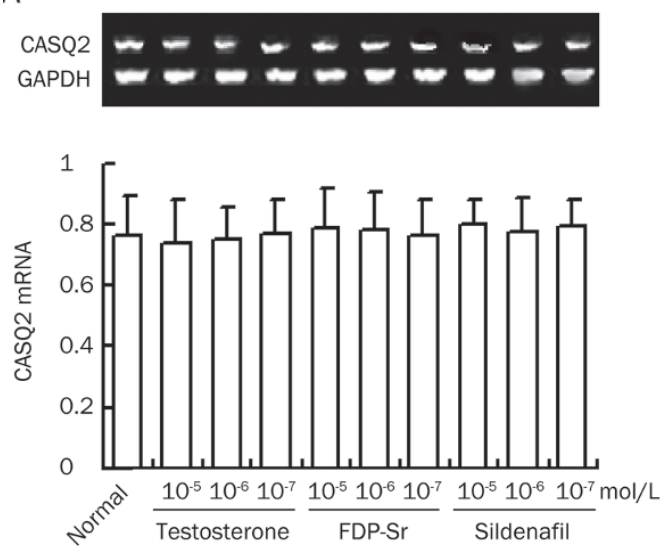

B
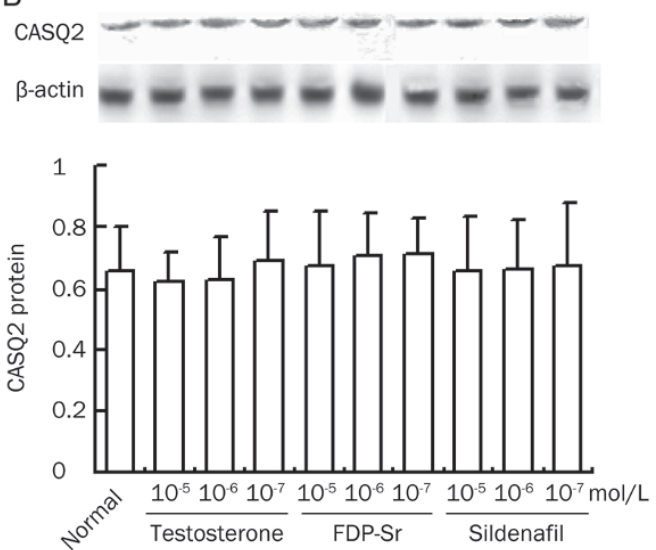

Figure 7. The $\mathrm{mRNA}$ and protein expression of CASQ 2 in the absence of diabetes mellitus with the three compounds in cardiomyocytes. (A) CASQ2 mRNA. (B) CASQ2 protein. Mean \pm SD. $n=10$. oxidase and induced NOS (iNOS) activity, which have been implicated in diabetic insults ${ }^{[26]}$. Increased ROS disturb many of the normal functions of the ER, induce cellular damage, and lead to apoptosis ${ }^{[27]}$. Activated iNOS can be combined with $\mathrm{NO}$ to form toxic peroxynitrite $(\mathrm{ONOO} \cdot-)$, which impairs the subcellular apparatus of the myocardium. Upregulation of iNOS is associated with xanthine oxidase activation and upregulation of NADPH oxidase, p22phox, p40phox, and p47phox, leading to more cardiac dysfunction in diabetic cardiomyopathy ${ }^{[28]}$. These changes in calcium homeostasis and oxidative stress are consistent with our previous findings ${ }^{[8,9]}$. In addition, oxidants and inflammatory cytokines, such as ROS, iNOS, endothelin-1, and leptin, damage DNA, leading to myocardial cell death.

Diabetes is often accompanied by obesity, abnormal lipid metabolism, and leptin upregulation ${ }^{[29,30]}$. Leptin is a peptide 
hormone secreted by fat cells that stimulates the satiety center in the hypothalamus. However, in pathological conditions, prolonged leptin upregulation eventually leads to leptin resistance and increased expression of the leptin receptor OBRb. $\mathrm{OBRb}$ serves as an inflammatory factor that mediates myocardial injury ${ }^{[22]}$.

Disorder of calcium-modulating proteins, such as FKBP12.6 and SERCA2a, has been shown to play a role in diabetic or isoproterenol-induced cardiomyopathy, which causes oxidative stress and inflammation ${ }^{[8,12,21]}$. Downregulation of FKBP12.6 and SERCA2a leads to more intracellular $\mathrm{Ca}^{2+}$ at the end of diastole, known as calcium leak, which we found in L-thyroxine-induced cardiomyopathy ${ }^{[30]}$. Thus, downregulation of FKBP12.6 is a marker of calcium leak, which causes cardiac dysfunction and severe arrhythmias. Endothelin receptor antagonists and CPU86017, a berberine derivative, relieve these conditions ${ }^{[23,30]}$.

Mutation of CASQ2 may cause an autosomal recessive form of the life-threatening arrhythmia CPVT. Additionally, mutations of RyR2 can lead to an autosomal dominant form of $\mathrm{CPVT}^{[25]}$. Normally, CASQ2 and RyR2 form tetramers. When SR calcium is low, RyR2 places the tetramer in an inhibitory state. When SR calcium increases, the CASQ2-RyR2 complex is weakened. If CASQ2 is mutated, the tetramer is even more unstable, and RyR2 sensitivity to calcium increases. As a result, SR calcium leak was more likely ${ }^{[31,32]}$. We found that downregulation of CASQ2 is an important event in diabetic cardiomyopathy. Also, downregulation of FKBP12.6 and SERCA2a are likely involved. For example, an increase in the cytosol:SR calcium ratio disrupts SERCA2a function. Therefore, concentrations of calcium in the cytoplasm increase, and excessive calcium flows through sodium calcium exchanger (NCX), leading to increased $\mathrm{Na}^{+}$influx into myocardial cells, delayed repolarization, and arrhythmias ${ }^{[33]}$. Interestingly, in addition to mutation, CASQ2 can be altered by posttranscriptional modifications due to either isoproterenol or hyperglycemia, which also lead to cardiac failure and tachyarrhythmias. CASQ2, FKBP12.6, and SERCA2a are sensitive markers of oxidative stress in the myocardium. Isoproterenol-induced downregulation of FKBP12.6, SERCA2a, and CASQ2 has been associated with increased $\operatorname{ROS}^{[34,35]}$. ROS produced by hyperglycemia and AGEs are considered to be mediated by the same mechanism as the downregulation of FKBP12.6, SERCA2a, and CASQ2 in diabetic cardiomyopathy. Therefore, sildenafil and FDP-Sr were effective in blunting the abnormalities in calcium-handling proteins and proinflammatory cytokines, such as leptin, iNOS, and endothelin-1, through their antioxidant activities. As demonstrated in this study, changes in CASQ2, FKBP12.6, and SERCA2a are not affected by exogenous testosterone even though the low serum androgen levels were corrected. This is likely due to the mild antioxidant activity of testosterone.

Myocardial diacylglycerol (DAG) activates protein kinase C (PKC) signaling pathways. This process has been found in the vascular tissue of diabetic animals and leads to increased blood vessel susceptibility to hyperglycemia. The PKC/
$\mathrm{NF \kappa B} / \mathrm{c}$-fos pathway is activated in neonatal rat cardiomyocytes in high-glucose medium ${ }^{[36]}$. Activated PKC $\varepsilon$ has been found in isoproterenol-induced cardiomyopathy and in the vasculature of STZ-injected rats ${ }^{[37,38]}$. PKCE overexpression may participate in downstream events, including $\mathrm{K}^{+}$channel and calcium-handling protein abnormalities, that are involved in diabetic or isoproterenol-induced cardiomyopathy ${ }^{[9,12,35]}$. In this study, PKC $\varepsilon$ mRNA expression was elevated, and $\mathrm{pPKC} \varepsilon$ was increased in association with cardiac dysfunction. FDP-Sr and sildenafil decreased PKC $\varepsilon$ mRNA and $\mathrm{pPKC} \varepsilon$ protein significantly, likely through their antioxidant activity .

\section{Conclusion}

In this study, CASQ2 was found to be abnormally expressed in diabetic cardiomyopathy. CASQ2 is actively involved in the pathogenesis of diabetic cardiomyopathy and is closely related to oxidative stress and inflammation. Furthermore, other calcium-handling proteins, such as SERCA2a and FKBP12.6, were abnormally expressed. Oxidants, iNOS, OBRb, and pPKC $\varepsilon$ serve as markers of the metabolic changes responsible for inducing diabetic cardiomyopathy. Sildenafil and FDP-Sr are beneficial for treating diabetic cardiomyopathy through their antioxidant and anti-inflammatory properties. However, FDP-Sr and sildenafil did not affect blood glucose or lipid metabolism, leaving the abnormal metabolic changes intact. Testosterone did not improve cardiac dysfunction or calcium homeostasis in the myocardium.

\section{Acknowledgements}

This project was supported by the National Natural Science Foundation of China (№ 30873112).

\section{Author contribution}

Yu-si CHENG conducted the project, processed the data, and wrote the manuscript. Qi ZHANG provided the sildenafil. Hui JI assisted with the project and discussion of the manuscript. De-zai DAI and Yin DAI designed the hypothesis and project and revised the manuscript.

\section{References}

1 Lebeche D, Davidoff AJ, Hajjar RJ. Interplay between impaired calcium regulation and insulin signaling abnormalities in diabetic cardiomyopathy. Nat Clin Pract Cardiovasc Med 2008; 5: 715-24.

2 Aneja A, Tang WH, Bansilal S, Garcia MJ, Farkouh ME. Diabetic cardiomyopathy: insights into pathogenesis, diagnostic challenges, and therapeutic options. Am J Med 2008; 121: 748-57.

3 Asghar O, Al-Sunni A, Khavandi K, Khavandi A, Withers S, Greenstein A, et al. Diabetic cardiomyopathy. Clin Sci (Lond) 2009; 116: 741-60.

4 Montagnani M. Diabetic cardiomyopathy: how much does it depend on AGE? Br J Pharmacol 2008; 154: 725-26.

5 Tang $\mathrm{M}$, Zhong $\mathrm{M}$, Shang $\mathrm{Y}$, Lin $\mathrm{H}$, Deng J, Jiang $\mathrm{H}$, et al. Differential regulation of collagen types I and III expression in cardiac fibroblasts by AGEs through TRB3/MAPK signaling pathway. Cell Mol Life Sci 2008; 65: 2924-32.

6 Hayat SA, Patel B, Khattar RS, Malik RA. Diabetic cardiomyopathy: mechanisms, diagnosis and treatment. Clin Sci (Lond) 2004; 107: 539-57. 
care physician. Can J Urol 2008; 15: 71-7.

7 Bidasee KR, Zhang Y, Shao CH, Wang M, Patel KP, Dincer UD, et al. Diabetes increases formation of advanced glycation end products on Sarco(endo)plasmic reticulum $\mathrm{Ca}^{2+}$-ATPase. Diabetes 2004; 53: 463-73.

8 Qi MY, Xia HJ, Dai DZ, Dai Y. A novel endothelin receptor antagonist CPU0213 improves diabetic cardiac insufficiency attributed to upregulation of the expression of FKBP12.6, SERCA2a, and PLB in rats. J Cardiovasc Pharmacol 2006; 47: 729-35.

9 Qi MY, Liu HR, Dai DZ, Li N, Dai Y. Total triterpene acids, active ingredients from Fructus Corni, attenuate diabetic cardiomyopathy by normalizing ET pathway and expression of FKBP12.6 and SERCA2a in streptozotocin-rats. J Pharm Pharmacol 2008; 60: 1687-94.

10 Boudina S, Abel ED. Diabetic cardiomyopathy revisited. Circulation 2007; 115: 3213-23.

11 Györke S. Molecular basis of catecholaminergic polymorphic ventricular tachycardia. Heart Rhythm 2009; 6: 123-9.

12 Li N, Jia N, Dai DZ, Hu C, Dai Y. Role of endothelin in the effects of isoprenaline on potassium currents and calsequestrin 2 expression in the heart. Clin Exp Pharmacol Physiol 2010; 37: 557-63.

13 Maggio M, Basaria S. Welcoming low testosterone as a cardiovascular risk factor. Int J Impot Res 2009; 21: 261-4.

14 Jones TH. Testosterone deficiency: a risk factor for cardiovascular disease? Trends Endocrinol Metab 2010; 21: 496-503.

15 Francomano D, Bruzziches R, Natali M, Aversa A, Spera G. Cardiovascular effect of testosterone replacement therapy in aging male. Acta Biomed 2010; 81: 101-6.

16 Mancini A, Leone E, Festa R, Grande G, Silvestrini A, de Marinis L, et al. Effects of testosterone on antioxidant systems in male secondary hypogonadism. J Androl 2008; 29: 622-9.

17 Montani D, Chaumais MC, Savale L, Natali D, Price LC, Jaïs X, et al. Phosphodiesterase type 5 inhibitors in pulmonary arterial hypertension. Adv Ther 2009; 26: 813-25.

18 Guazzi M. Sildenafil and phosphodiesterase-5 inhibitors for heart failure. Curr Heart Fail Rep 2008; 5: 110-4.

19 Zhang Q, Liu HR, Ying HJ, Dai DZ, Tang XY, Dai Y. Strontium fructose 1,6-diphosphate early diabetic testopathy by suppressing abnormal testicular matrix metalloproteinase system in streptozocin-treated rats. J Pharm Pharmacol 2009; 61: 229-36.

20 Xu M, Dai DZ, Zhang Q, Cheng YS, Dai Y. Upregulated NADPH oxidase contributes to diabetic testicular complication and is relieved by strontium fructose 1,6-diphosphate. Exp Clin Endocrinol Diabetes 2010; 118: 459-65.

21 Cheng YS, Dai DZ, Dai Y. Stress-induced cardiac insufficiency relating to abnormal leptin and FKBP12.6 is ameliorated by CPU0213, an endothelin receptor antagonist, which is not affected by the CYP3A suppressing effect of erythromycin. J Pharm Pharmacol 2009; 61: 569-76.

22 Na T, Dai DZ, Tang XY, Dai Y. Upregulation of leptin pathway correlates with abnormal expression of SERCA2a, phospholamban and the endothelin pathway in heart failure and reversal by CPU86017. Naunyn Schmiedebergs Arch Pharmacol 2007; 375: 39-49.

23 Casey RW, Barkin J. Testosterone replacement therapy for the primary
24 Bilginoglu A, Seymen A, Tuncay E, Zeydanli E, Aydemir-Koksoy A, Turan B. Antioxidants but not doxycycline treatments restore depressed beta-adrenergic responses of the heart in diabetic rats. Cardiovasc Toxicol 2009; 9: 21-9.

25 Adeghate E. Molecular and cellular basis of the aetiology and management of diabetic cardiomyopathy: a short review. Mol Cell Biochem 2004; 261: 187-91.

26 Violi F, Basili S, Nigro C, Pignatelli P. Role of NADPH oxidase in atherosclerosis. Future Cardiol 2009; 5: 83-92.

27 Münzel T. Endothelial dysfunction: pathophysiology, diagnosis and prognosis. Dtsch Med Wochenschr 2008; 133: 2465-70.

28 Rajesh M, Mukhopadhyay P, Bátkai S, Mukhopadhyay B, Patel V, Haskó G, et al. Xanthine oxidase inhibitor allopurinol attenuates the development of diabetic cardiomyopathy. J Cell Mol Med 2009; 13: 2330-41.

29 Iciek R, Wender-Ozegowska E, Seremak-Mrozikiewicz A, Drews K, Brazert J, Pietryga M. Leptin gene, leptin gene receptor polymorphisms and body weight in pregnant women with type 1 diabetes mellitus. Ginekol Pol 2008; 79: 592-601.

30 Zhang Y, Huang ZJ, Dai DZ, Feng Y, Na T, Tang XY, et al. Downregulated FKBP12.6 expression and upregulated endothelin signaling contribute to elevated diastolic calcium and arrhythmogenesis in rat cardiomyopathy produced by L-thyroxin. Int J Cardiol 2008; 130: 463-71.

31 Liu N, Rizzi N, Boveri L, Priori SG. Ryanodine receptor and calsequestrin in arrhythmogenesis: what we have learnt from genetic diseases and transgenic mice. J Mol Cell Cardiol 2009; 46: 149-59.

32 Györke S, Hagen BM, Terentyev D, Lederer WJ. Chain-reaction $\mathrm{Ca}^{2+}$ signaling in the heart. J Clin Invest 2007; 117: 1758-62.

33 Song L, Alcalai R, Arad M, Wolf CM, Toka O, Conner DA, et al. Calsequestrin 2 (CASQ2) mutations increase expression of calreticulin and ryanodine receptors, causing catecholaminergic polymorphic ventricular tachycardia. J Clin Invest 2007; 117: 1814-23.

34 Li N, Jia N, Dai DZ, Hu C, Dai Y. Role of endothelin in the effects of isoprenaline on potassium currents and calsequestrin 2 expression in the heart. Clin Exp Pharmacol Physiol 2010; 37: 557-63

35 Li N, Jia N, Dai DZ, Dai Y. Endothelin receptor antagonist CPU0213 and vitamin E reverse downregulation of FKBP12.6 and SERCA2a: a role of hyperphosphorylation of PKCepsilon. Eur J Pharmacol 2008; 591: 211-8.

36 Min W, Bin ZW, Quan ZB, Hui ZJ, Sheng FG. The signal transduction pathway of PKC/NF-kappaB/c-fos may be involved in the influence of high glucose on the cardiomyocytes of neonatal rats. Cardiovasc Diabetol 2009; 8: 8.

37 Cheng YS, Dai DZ, Dai Y. Isoproterenol disperses distribution of NADPH oxidase, MMP-9, and pPKCepsilon in the heart, which are mitigated by endothelin receptor antagonist CPU0213. Acta Pharmacol Sin 2009; 30: 1099-106.

38 Zheng YF, Dai DZ, Dai Y. NaHS ameliorates diabetic vascular injury by correcting depressed connexin 43 and 40 in the vasculature in streptozotocin-injected rats. J Pharm Pharmacol 2010; 62: 615-21. 